\title{
Sur la rectification de quelques courbes.
}

\author{
(par le D.r J. Bootir, F. R. S., London).
}

I. Rectification de la courbe inverse de l'cllipse centrale.

Ce problème mórite d'ŝtro discuté à cause de l'ólégance remarquable de sa solution, qui dépend de l'évaluation d'une intégrale elliptique de troisième espèce à paramètre circulaire.

On dit que deux courbes sont inverses l'une de l'autre lorsque le produit de leurs rayons vecteurs superposés est constant, c'est-à-dire que:

Soit :

$$
R r=c^{2} \text {. }
$$

$$
\frac{x^{2}}{a^{2}}+\frac{y^{2}}{b^{2}}=1
$$

l'équation de l'ellipse, le centre ćtant au pôle, et soit $R r=k_{a} b$ : on aura, pour la courbe inverse, l'équation:

$$
k^{2}\left(a^{2} y^{2}+b^{2} x^{2}\right)=\left(x^{2}+y^{2}\right)^{2} .
$$

On peut simplifier la discussion, sans restreindra la généralité, en prenant $k=1$. L'équation de la courbe inverse à l'ellipse, lo centre étant au pôle, est alors:

Si l'on pose:

$$
a^{2} y^{2}+b^{2} x^{2}=\left(x^{2}+y^{2}\right)^{2} .
$$

cette équation devient:

$$
x=r \cos \phi, \quad y=r \sin \phi,
$$

$$
a^{2} \sin ^{2} \phi+b^{2} \cos ^{2} \phi=r^{2}
$$

d'où l'on tire, après quelques réductions simples,

$$
\frac{d s^{2}}{d \varphi^{2}}=\frac{a^{4} \sin ^{2} \varphi+b^{4} \cos ^{2} \varphi}{a^{2} \sin ^{2} \varphi+b^{2} \cos ^{2}{ }^{2} \varphi^{2}}
$$


La substitution:

$$
a^{2} \tan \phi=b^{2} \tan \lambda,
$$

change cette formule en la suivante:

$$
\frac{d s^{2}}{d \underline{p}^{2}}=\frac{a^{2} b^{2}}{a^{2} \cos ^{2} \lambda+b^{2} \sin ^{2} \lambda}
$$

ot puisque on en tire aussi:

$$
\frac{d \varphi}{d \lambda}=\frac{b^{2}}{a^{2}} \frac{\cos ^{2} \varphi}{\cos ^{2} \lambda} \text {, de mème que } \frac{\cos ^{2} \varphi}{\cos ^{2} \lambda}=\frac{a^{4}}{a^{4} \cos ^{2} \lambda+b^{4} \sin ^{2} \lambda},
$$

substituant et simplifiant on obtient:

$$
\frac{d s}{d \lambda}=\frac{a^{3} b^{3}}{\left(a^{4} \cos ^{2} \lambda+b^{4} \sin ^{2} \lambda\right) \sqrt{a^{2} \cos ^{2} \lambda+b^{2} \sin ^{2} \lambda}},
$$

ou :

$$
\frac{d s}{d \lambda}=\frac{b^{3}}{a^{2}} \frac{1}{\left[1-\left(\frac{a^{4}-b^{4}}{a^{4}}\right) \sin ^{2} \lambda\right] \sqrt{1-\left(\frac{a^{2}-b^{2}}{a^{2}}\right) \sin ^{2} \lambda}}
$$

Faisant:

$$
\frac{a^{2}-b^{2}}{a^{2}}=c^{2} \quad \text { et } \frac{a^{4}-b^{4}}{a^{4}}=m
$$

on $\mathbf{a}$ :

$$
m=\frac{a^{2}+b^{2}}{a^{2}} c^{2}, \quad m>c^{2}
$$

et par suite, intégrant:

$$
s=\frac{b^{3}}{a^{2}} \int \frac{d \lambda}{\left[1-m \sin ^{2} \lambda\right]} \frac{\sqrt{1-c^{2} \sin ^{2} \lambda}}{\sqrt{1-},}
$$

intégrale elliptique de troisième espèce à paramètre circulaire, car $m>c^{2}$.

Imaginons le cylindre droit dont la base est l'ellipse aux demi-axes $a$ et $b$, et la sphère décrite du centre avec un rayon $=\sqrt{a^{2}+b^{2}}$. Cette sphère coupo le cylindre suivant une ellipse sphérique.

Soient $\alpha$ ct $\beta$ les demi-angles principaux de cette ellypse sphérique, alors:

$$
\sin ^{2} \alpha=\frac{a^{2}}{a^{2}+b^{2}}, \sin ^{2} \beta=\frac{b^{2}}{a^{2}+b^{2}}, \tan ^{2} \alpha=\frac{a^{2}}{b^{2}}, \tan ^{2} \beta=\frac{b^{2}}{a^{2}} .
$$

D'ici on tire:

$$
\frac{a^{4}-b^{4}}{a^{4}}=\frac{\tan ^{2} \alpha-\tan ^{2} \beta}{\tan ^{2} \alpha}, \frac{a^{2}-b^{2}}{a^{2}}=\frac{\sin ^{2} \alpha-\sin ^{2} \beta}{\sin ^{2} \alpha},
$$


ct:

$$
\frac{b^{3}}{a^{2}}=\sqrt{a^{2}+b^{2}} \frac{\operatorname{lan} \theta}{\tan \alpha} \sin \beta
$$

Effectuant ces substitutions dans l'équation (8) il vient:

$$
\frac{s}{\sqrt{a^{2}+b^{2}}}=\frac{\tan \beta}{\tan \alpha} \sin \beta \int \frac{d \lambda}{\left[1-\left(\frac{\tan ^{2} \alpha-\tan ^{2} \beta}{\tan ^{2} \alpha}\right) \sin ^{2} \lambda\right] \sqrt{1-\left(\frac{\sin ^{2} \alpha-\sin ^{2} \bar{\beta}}{\sin ^{2} \alpha}\right) \sin ^{2} \lambda}} .
$$

Or dans les Philosophical Transactions pour 1852, Part II, p. 319, j'ai montré que l'expression d'un are de l'ellipse sphérique qui résulte de l'interscction d'un cône aux demi-angles $\alpha$ et $\beta$ avec une sphéro concentrique est donnée par la formule:

$$
\sigma=\frac{\tan \beta}{\tan \gamma} \sin \beta \int \frac{d \varphi}{\left[1-\left(\frac{\tan ^{2} \alpha-\tan ^{2} \beta}{\tan ^{2} \alpha}\right) \sin ^{2} \phi\right] V} \frac{d-\left(\frac{\sin ^{2} \alpha-\sin ^{2} \beta}{\sin ^{2} \alpha}\right) \sin ^{2} \phi}{}
$$

Soit $e$ l'exentricité de la basc plane du cône, savoir $e^{2}=\frac{a^{2}-b^{2}}{a^{2}}, 2 \varepsilon$ l'angle des deux lignes focales, et $2 \eta$ l'angle des dẹx axes cycliques, c'està-dire des deux droites normales aux sections circulairos du côno; d'apres le même Mémoiro on aura:

$$
c^{2}=\frac{\tan ^{2} \alpha-\tan ^{2} \beta}{\tan ^{2} \alpha}, \quad \sin ^{2} \eta=\frac{\sin ^{2} \alpha-\sin ^{2} \beta}{\sin ^{2} \gamma},
$$

et:

$$
\tan ^{2} \varepsilon=\frac{\sin ^{2} \alpha-\sin ^{2} \beta}{\cos ^{2} \alpha}
$$

et la précédente expression de l'arc de l'ellipse sphérique deviendra:

$$
\sigma=\frac{\tan \beta}{\tan \alpha} \sin \beta \int \frac{d \varphi}{\left[1-e^{2} \sin ^{2} \varphi\right] \sqrt{1-\sin ^{2} n \cdot \sin ^{2} \varphi}} .
$$

Désignant par $m$ et $n$ deux paramètres conjugués, on a:

$$
(1-m)(1+n)=1-c^{2},
$$

ainsi qu'on peut le voir dans tout ouvrage élémentaire sur les intégrales elliptiques; donc si l'on fait $m=e^{2}$ et $c^{2}=\sin ^{2} \eta$, on a $n=\frac{\sin ^{2} \alpha-\sin ^{2} \beta}{\cos ^{2} \alpha}$ ou :

$$
n=\tan ^{2} \varepsilon \text {. }
$$


Les trois quantités: $e$ excentricité de la base du cône, $2 \varepsilon$ angle des lignes focales et $2 \eta$ angle des lignes cycliques, sont liées par l'équation simple:

$$
1-e^{2}=\cos ^{2} \eta \cdot \cos ^{2} \varepsilon
$$

Le coefficient de l'intégrale (12), c'est-à-dire $\frac{\tan \beta}{\tan \%} \sin \beta$, est ce qu'on nomme quclquefois le criterium de circularité, car:

$$
\frac{\tan ^{2} \beta}{\tan ^{2} \alpha} \sin ^{2} \beta=(1-i n)\left(1-\frac{c^{2}}{m}\right)
$$

par suite on peut écrire:

$$
\sigma=\sqrt{(1-m)\left(1-\frac{c^{2}}{n}\right)} \int \frac{d \varphi}{\left[1-m \sin ^{2} \varphi\right] \sqrt{1-c^{2} \sin ^{2} \varphi}} .
$$

Notre conique sphérique a ses arcs principaux supplémentaires, car puisque $\tan \alpha=\frac{a}{b}, \tan \beta=\frac{b}{a}$, on a $\tan \alpha \cdot \tan \beta=1$ d'où $\alpha+\beta=\frac{\pi}{2} \cdot$.llc est égale à sa réciproque tournée d'un anģle droit. Les axes focaux de l'une sont les axes cycliques de l'autre: par conséquent la rectification de l'une dépend de la quadrature de l'autre, ainsi qu'on peut le voir dans le Mémoire cité ci-dessus.

II. De la rectification de la courbe représentée par l'équation:

$$
a^{2} x^{2}-b^{2} y^{2}=\left(x^{2}+y^{2}\right)^{2}
$$

Posant $x=r \sin \phi, y=r \cos \phi$, il vient:

$$
a^{2} \sin ^{2} \phi-b^{2} \cos ^{2} \hat{\phi}=r^{2},
$$

d'où :

$$
\frac{d s^{2}}{d \varphi^{2}}=\frac{a^{4} \sin ^{2} \varphi+b^{4} \cos ^{2} \varphi}{a^{2} \sin ^{2} \varphi-b^{2} \cos ^{2} \varphi} .
$$

Prenant :

$$
a^{2} \tan ^{2} \phi=l^{2} \sec ^{2} \lambda
$$

et faisant les substitutions nécessaires, on trouvera:

$$
\frac{d s}{d \lambda}=\frac{a b \sqrt{a^{2}+b^{2} \cos ^{2} \lambda}}{b^{2}+a^{2} \cos ^{2} \lambda}
$$


ou :

$$
\frac{d s}{d \lambda}=\frac{a b\left[a^{2}+b^{2}-b^{2} \sin ^{2} \lambda\right]}{\left[a^{2}+b^{2}-a^{2} \sin ^{2} \lambda\right] \sqrt{a^{2}+b^{2}-b^{2} \sin ^{2} \lambda}}
$$

ou encole:

$$
\frac{d s}{d \lambda}=\frac{a b}{\sqrt{a^{2}+b^{2}}} \cdot \frac{\left[1-\frac{b^{2}}{a^{2}-1-b^{2}} \sin ^{2} \lambda\right]}{\left[1-\frac{a^{2}}{a^{2}+b^{2}} \sin ^{2} \lambda\right] \sqrt{1-\frac{b^{2}}{a^{2}+b^{2}} \sin ^{2} \lambda}},
$$

ou enfin:

$$
\frac{d s}{d \lambda}=\frac{b^{3}}{a \sqrt{a^{2}+b^{2}}} \cdot \frac{\left[\frac{a^{2}}{b^{2}}-1+1-\frac{a^{2}}{a^{2}+b^{2}} \sin ^{2} \lambda\right]}{\left[1-\frac{a^{2}}{a^{2}+b^{2}} \sin ^{2} \lambda\right] \sqrt{1-\frac{b^{2}}{a^{2}+b^{4}} \sin ^{2} \lambda}} \cdot
$$

En faisant:

$$
\frac{a^{2}}{a^{2}+b^{2}}=m, \quad \frac{b^{2}}{a^{2}+b^{2}}=c^{2},
$$

la dernière équation devient:

$$
\frac{d s}{d \lambda}=\frac{b\left(a^{2}-b^{2}\right)}{a \sqrt{a^{2}+b^{2}}} \frac{1}{\left[1-m \sin ^{2} \lambda\right] \sqrt{1-c^{2} \sin ^{2} \lambda}}+\frac{b^{3}}{a \sqrt{a^{2}+b^{2}}} \cdot \frac{1}{\sqrt{1-c^{2} \sin ^{2} \lambda}},
$$

ou, intégrant:

$$
s=\frac{b}{a} \frac{a^{2}-b^{2}}{\sqrt{a^{2}+b^{2}}} \int \frac{d \lambda}{\left[1-m \sin ^{2} \lambda\right] \sqrt{1-c^{2} \sin ^{2} \lambda}}+\frac{b^{3}}{a \sqrt{a^{2}+b^{2}}} \int \frac{d \lambda}{\sqrt{1-c^{2} \sin ^{2} \lambda}} \cdot
$$

Si la courbe est la lemniscate, on a $a=b$, et cette expression devient:

$$
s=\frac{a}{\sqrt{2}} \int \frac{d \lambda}{\sqrt{1-\frac{1}{2} \sin ^{2} \lambda}} \cdot
$$

III. De la rectification de la courbe d'intersection d'un cylindre hyperbolique avec une sphère concentrique.

L'axe du cylindre hyperbolique soit dirigé suivant l'axe des $y$, et soient:

$$
\frac{z^{2}}{a^{2}}-\frac{x^{2}}{b^{2}}=1, \quad x^{2}+y^{2}+z^{2}=r^{2}
$$

les équations du cylindre et do la sphère concentrique. Donc:

$$
a^{2} x^{2}=b^{2} z^{2}-a^{2} b^{2}, \quad a^{2} y^{2}=a^{2}\left(b^{2}+r^{2}\right)-\left(a^{2}+b^{2}\right) z^{2} .
$$




\section{Posant :}

$$
z^{2}=a^{2} \cos ^{2} \phi+a^{2} \frac{b^{2}+r^{2}}{a^{2}+b^{2}} \sin ^{2} \phi
$$

il vient:

$$
x^{2}=\frac{b^{2}\left(r^{2}-a^{2}\right)}{a^{2}+b^{2}} \sin ^{2} \phi, \quad y^{2}=\left(r^{2}-a^{2}\right) \cos ^{2} \phi
$$

d'où :

$$
\frac{d x^{2}}{d \varphi^{2}}=\frac{l^{2}\left(r^{2}-a^{2}\right)}{a^{2}+b^{2}} \cos ^{2} \phi, \quad \frac{d y^{2}}{d \vartheta^{2}}=\left(r^{2}-a^{2}\right) \sin ^{2} \phi
$$

et

$$
\frac{d z^{2}}{d \tau^{2}}=\frac{a^{2}\left(r^{2}-a^{2}\right)^{2} \sin ^{2} \varphi \cos ^{2} \varphi}{\left(a^{2}+b^{2}\right)\left[\left(a^{2}+b^{2}\right) \cos ^{2} \varphi+\left(b^{2}+r^{2}\right) \sin ^{2} \varphi\right]}
$$

On en conclut:

$$
\frac{1}{\sqrt{r^{2}-a^{2}}} \frac{d s}{d i}=\sqrt{\frac{b^{2}+r^{2} \sin ^{2} \varphi}{\left(a^{2}+b^{2}\right)+\left(r^{2}-a^{2}\right) \sin ^{2} \varphi}}
$$

La substitution:

$$
\tan ^{2} \phi=\frac{b^{2}}{b^{2}+r^{2}} \tan ^{2} \lambda
$$

et l'intégration subséquente donnent:

$$
s=\frac{b^{2} \sqrt{r^{2}-a^{2}}}{\sqrt{a^{2}+b^{2}} \sqrt{b^{2}+r^{2}}} \int \frac{d \lambda}{\left[1-\frac{r^{2}}{b^{2}+r^{2}} \sin ^{2} \lambda\right] \sqrt{1-\frac{a^{2}}{a^{2}+b^{2}} \sin ^{2} \lambda}} .
$$

Maintenant si l'on fait :

et

$$
\frac{r^{2}}{b^{2}+r^{2}}=\frac{\tan ^{2} \theta-\tan ^{2} \rho}{\tan ^{2} \%}
$$

$$
\frac{a^{2}}{a^{2}+b^{2}}=\frac{\sin ^{2} \alpha-\sin ^{2} \beta}{\sin ^{2} \alpha}
$$

$\alpha$ et $\beta$ étant les demi-angles de la ligne d'intersection du cylindre hyperbolique avec la sphère, on aura:

$$
\frac{s}{r}-\frac{\tan \beta}{\tan \alpha} \sin \beta \int \frac{d \lambda}{\left[1-\left(\frac{\tan ^{2} \alpha-\tan ^{2} \beta}{\tan ^{2} \alpha}\right) \sin ^{2} \lambda\right] \sqrt{1-\left(\frac{\sin ^{2} \alpha-\sin ^{2} \beta}{\sin ^{2} \alpha}\right) \sin ^{2} \lambda}},
$$

ce qui est l'expression de l'arc do conique sphérique dont $2 \alpha$ et $2 \beta$ sont les arcs principaux. Voyez les Phil. Trans., même Mémoire, p. 319. 
De (36) on tire:

$$
\cos ^{2} \alpha=\frac{a^{2}}{r^{2}}, \quad \cos ^{2} \beta=\frac{a^{2}\left(b^{2}+r^{2}\right)}{r^{2}\left(a^{2}+b^{2}\right)} .
$$

Or dans le même Mémoire, pp. 316,317 , j’ai démontré que si $2 \varepsilon$ est l'angle des lignes focales du cône, $2 \eta$ l'angle des sections circulaires, et $e$ l'excentricité de la section plane normale à l'axe du cône, on a :

$$
\cos \varepsilon=\frac{\cos \alpha}{\cos \beta}, \quad \cos \eta=\frac{\sin \beta}{\sin \alpha}, \quad \text { et } e^{2}=\frac{\tan ^{2} \alpha-\tan ^{2} \beta}{\tan ^{2} \alpha} .
$$

Faisant ces substitutions dans (37) on obtient ce résultat:

$$
\frac{s}{r}=\sigma=\frac{\tan \beta}{\tan \alpha} \sin \beta \int \frac{d \lambda}{\left[1-e^{2} \sin ^{2} \lambda\right] \sqrt{1-\sin ^{2} \eta \cdot \sin ^{2} \lambda}},
$$

dont la forme est la plus simple à laquelle l'intégrale elliptique de troisième espèce à paramètre circulaire puisse ctre ramenée: $\sigma$ est l'arc de la courbe semblable au rayon 1 .

Comme on a:

$$
\sin ^{2} n=\frac{a^{2}}{a^{2}+b^{2}}
$$

valeur indépendante de $r$, nous pouvons dire que si des sphères concentriques en nombre quelconque coupent un même cylindre hyperbolique, les cônes qui passent par les différentes courbes d'intersection ont tuntes leurs sections circulaires paralleles entr'elles.

Soit $\nu$ l'angle que les plans asymptotiques du cylindre hyperbolique font avec le plan des $x y$. On aura:

$$
\tan \nu=\frac{a}{b} \text { et } \sin ^{2} \nu=\frac{a^{2}}{a^{2}+b^{2}},
$$

d'où $v=\eta$ C'est-à-dire que: les sections circulaires du cône sont paralleles aux plans asymptotiques du cylindre hyperbolique.

IV. Au lieu de regarder le rayon de la sphère (29) comme une quantité arbitraire, prenons:

$$
r^{2}=a^{2}+a \sqrt{a^{2}+b^{2}}, \text { et } j=\frac{\sqrt{a^{2}+b^{2}}-a}{\sqrt{a^{2}+b^{2}}+a} .
$$


Effectuant les réductions et les substitutions nécessaires dans (36), on obtient :

$$
\frac{s}{r}=\sigma=\frac{2 j}{1+j} \int \frac{d \lambda}{\left[1-\left(\frac{1-j}{1+j}\right) \sin ^{2} \lambda\right] \sqrt{1-\left(\frac{1-j}{1+j}\right)^{2} \sin ^{2} \lambda}} .
$$

Or comme dans ce cas particulier le paramètre est égal à la racine carrée du module, savoir $m=c$, on sait que l'intégrale elliptique peut être ramenée à celle de la première espèce, et la courbe rentre dans cette espèce particulière de coniques sphériques que j'ai nommée parabole sphérique, dans le Mémoire cité plusieurs fois. A la page 332 j'ai montré que:

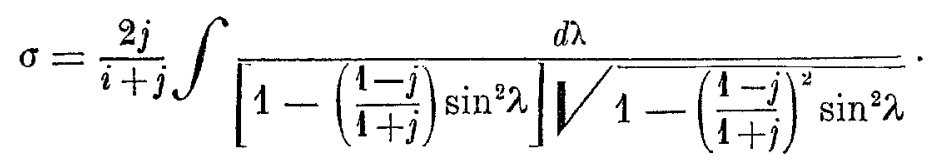

et:

$$
\sigma=j \int \frac{d \mu}{\sqrt{1-i^{2} \sin ^{2} \mu}}+\tan ^{-1}\left[\frac{j \tan \mu}{\sqrt{1-i^{2} \sin ^{2} \mu^{2}}}\right]
$$

étant:

$$
i^{2}+j^{2}=1 \text {, et } \tan (\lambda-\mu)=j \tan \mu \text {. }
$$

Egalant les valeurs de $\sigma$ on trouve:

$$
\left.\begin{array}{l}
\frac{2 j}{1+j} \int \frac{d \lambda}{\left[1-\left(\frac{1-j}{1 j}\right) \sin ^{2} \lambda\right] \sqrt{1-\left(\frac{1-j}{1+j}\right)^{2} \sin ^{2} \lambda}}, \\
=j \int \frac{d \mu}{\sqrt{1-i^{2} \sin ^{2} j,}}+\tan ^{-1}\left[\frac{j \tan \psi}{\sqrt{1-i^{2} \sin ^{2} j \cdot}}\right] .
\end{array}\right\}
$$

Cette rélation a déjà été établie par Lagraxae à l'aide de considerations d'une nature purement analytique. 